\title{
Robust Stabilization for Stochastic Systems with Time-Delay and Nonlinear Uncertainties
}

\author{
Zhiguo Yan, ${ }^{1}$ Guoshan Zhang, ${ }^{2}$ and Xingguang $Q i^{1}$ \\ ${ }^{1}$ School of Electrical Engineering and Automation, Shandong Polytechnic University, Jinan 250353, China \\ ${ }^{2}$ School of Electrical Engineering and Automation, Tianjin University, Tianjin 300072, China
}

Correspondence should be addressed to Zhiguo Yan, yanzg500@sina.com

Received 17 September 2011; Accepted 3 November 2011

Academic Editor: Weihai Zhang

Copyright (C) 2012 Zhiguo Yan et al. This is an open access article distributed under the Creative Commons Attribution License, which permits unrestricted use, distribution, and reproduction in any medium, provided the original work is properly cited.

This paper deals with the problem of robust stabilization of stochastic systems with time-delay and nonlinear uncertainties via memory state feedback. Based on Lyapunov krasoviskii functional, some sufficient conditions on local (global) stabilization are given in terms of matrix inequalities. In particular, these stabilizable conditions for a class of nonlinear stochastic time-delay systems are derived in the form of linear matrix inequalities, which have the advantage of easy computation. Moreover, the corresponding results are further extended to the stochastic multiple time-delays systems. Finally, an example is presented to show the superiority of memory state feedback controller to memoryless state feedback controller.

\section{Introduction}

Stochastic differential delay equations are one of the most useful stochastic models in applications, for example, aircraft, chemical or process control system, and distributed networks. It is known that time-delay is, in many cases, a source of poor system performance or instability. Hence, the stability and stabilization of stochastic time delay systems have been recently attracting the attention of a number of researchers, see [1-18] and the references therein. In [2], the authors studied the stability of linear stochastic systems with uncertain time delay by generalized Riccati equation approach, while [3] extended the results of [2] to nonlinear case via linear matrix inequalities. Reference [4] investigated the problems of stabilization for a class of linear stochastic systems with norm-bounded uncertainties and state delay, and it developed two criteria for the stability analysis: delay-dependent and delay-independent. The memoryless nonfragile state feedback control law for nonlinear stochastic time-delay systems was designed in [5], in which new sufficient conditions for the existence of such controllers were presented based on the linear matrix inequalities approach. 
Reference [6] was concerned with the stability analysis and proposed improved delaydependent stability criteria for uncertain stochastic systems with interval time-varying delay. The study of exponential stability of stochastic delay-differential equations was discussed in [7-9]. The output feedback stabilization of stochastic nonlinear time-delay systems was investigated in [10], and some stabilization criterions for nonlinear stochastic time-delay systems with state and control-dependent noise were given in [11,12] by means of matrix inequalities.

We usually design memoryless state feedback controller for the stabilization of systems because of its advantage of easy implementation. However, its performance, for time-delay systems, cannot be better than a memory state feedback controller which utilizes the available information of the size of delay. Reference [19] has given a general form of a memory state feedback (delayed feedback) controller:

$$
u(t)=G x(t)+\int_{t-\tau}^{t} G_{1}(s) x(s) d s
$$

But the task of storing all the previous states $x(\cdot)$ and computing the values of time-varying gain matrices $G_{1}(\cdot)$ makes the practical realization of infinite-dimensional controller (1.1) very difficult. For these reasons, the controller

$$
u(t)=G x(t)+G_{2} x(t-\tau)
$$

could be considered as a compromise between the performance improvement and the implementation simplicity. Reference [20] gave the sufficient conditions for the stabilization of deterministic state-delayed systems. References [21] and [22] designed a memory state feedback controller for neutral time-delay systems and singular timedelay systems, respectively. Reference [23] studied the stabilization problem for a class of discrete-time Markovian jump linear systems with time-delays both in the system state and in the mode signal via time-delayed controller and obtained a sufficient condition. What [13] actually studied is the stabilization problem of linear stochastic time-delay systems using generalized Riccati equation method. Up to now, to the best of the authors' knowledge, the issue on memory state feedback stabilization of stochastic systems with time-delay and nonlinear uncertainties has not been fully investigated in previous literatures.

In this paper, we consider the problem on robust stabilization for stochastic systems with time-delay and nonlinear uncertainties via memory state feedback. This problem contains three inevitable aspects of practical application: timedelay, nonlinear uncertainties and more effective controller, which is more complex than the stabilization of pure stochastic systems via memoryless control. These complexities result in some difficulties of memory stabilizing controller design. By the Itô formula, mathematical expectation properties, and matrix transformation, some sufficient conditions are obtained on locally and globally asymptotic stabilization in probability by means of matrix inequalities. Especially for a class of nonlinear stochastic time-delay systems, a sufficient condition for the existence of memory state feedback stabilizing controller is obtained in terms of LMIs, which has the advantage of easy computation. Meanwhile, a memoryless state feedback controller is also given as a special case of memory state feedback controller. Moreover, the robust stabilization problem for stochastic multiple time-delays systems is further studied and a general sufficient condition is derived. 
The paper is organized as follows. Some preliminaries and problem formulations are presented in Section 2. In Section 3, main results are given. Section 4 presents one example to illustrate the effectiveness of our developed results. Section 5 concludes this paper.

Notation 1. $A^{\prime}$ : the transpose of matrix $A ; A \geq 0(A>0): A$ is positive semidefinite (positive definite) symmetric matrix; $I$ : identity matrix; $\|\cdot\|$ : Euclidean norm; $L_{\mathscr{q}}^{2}\left([0, \infty), \mathbf{R}^{l}\right)$ : space of nonanticipative stochastic process $y(t) \in \mathbf{R}^{l}$ with respect to an increasing $\sigma$-algebra $\mathscr{f}_{t}(t \geq 0)$ satisfying $E \int_{0}^{\infty}\|y(t)\|^{2} d t<\infty . I_{n \times n}: n \times n$ identity matrix.

\section{Preliminaries and Problem Statement}

Consider the following continuous nonlinear stochastic time-delay systems:

$$
\begin{aligned}
d x(t)= & \left(A x(t)+B x(t-\tau)+B_{1} u(t)+H_{0}(x(t), x(t-\tau), u(t))\right) d t \\
& +\left(C x(t)+D x(t-\tau)+D_{1} u(t)+H_{1}(x(t), x(t-\tau), u(t))\right) d w(t), \\
x(t)= & \varnothing(t) \in L^{2}\left(\omega, \mathcal{F}_{0}, C\left([-\tau, 0], R^{n}\right)\right), \quad t \in[-\tau, 0]
\end{aligned}
$$

where $x(t) \in \mathbf{R}^{n}$ and $u(t) \in \mathbf{R}^{m}$ are system state and control input, respectively; $w(t)$ is 1-dimensional standard Wiener process defined on the probability space $\left(\Omega, \mathcal{F}, \mathcal{F}_{t}, P\right)$ with $F_{t}=\sigma\{w(s): 0 \leq s \leq t\} ; H_{i}(0, \cdot \cdot \cdot)=0, i=0,1 ; A, B, B_{1}, C, D$, and $D_{1}$ are constant matrices; $\tau>$ 0 is a certain timedelay. Under very mild conditions on $H_{i}(0, \cdot, \cdot), i=0,1,(2.1)$ exists a unique global solution [1]. It should be pointed out that any general nonlinear stochastic system which is sufficiently differentiable can take the form of (2.1) via Taylor's series expansion at the origin.

Next, we give the following definitions essential for the paper.

Definition 2.1 (see [1]). System (2.1) with $u(t)=0$ is said to be stable in probability, if for any $\epsilon>0$,

$$
\lim _{x \rightarrow 0} P\left(\sup _{t \geq 0}\|x(t)\|>\epsilon\right)=0 .
$$

Additionally, if we also have

$$
\lim _{x_{0} \rightarrow 0} P\left(\lim _{t \rightarrow \infty} x(t)=0\right)=1
$$

then system (2.1) with $u(t)=0$ is said to be locally asymptotically stable in probability.

If (2.2) holds and

$$
P\left(\lim _{t \rightarrow \infty} x(t)=0\right)=1
$$

for all $x_{0} \in \mathbf{R}^{n}$, then system (2.1) with $u(t)=0$ is said to be globally asymptotically stable in probability. 
Definition 2.2. If there exists a constant memory state feedback control law

$$
u(t)=K_{1} x(t)+K_{2} x(t-\tau),
$$

such that the equilibrium point of the closed-loop system

$$
\begin{aligned}
d x(t)= & \left(\left(A+B_{1} K_{1}\right) x(t)+\left(B+B_{1} K_{2}\right) x(t-\tau)+H_{0}\left(x(t), x(t-\tau), K_{1} x(t)+K_{2} x(t-\tau)\right)\right) d t \\
& +\left(\left(C+D_{1} K_{1}\right) x(t)+\left(D+D_{1} K_{2}\right) x(t-\tau)\right. \\
& \left.+H_{1}\left(x(t), x(t-\tau), K_{1} x(t)+K_{2} x(t-\tau)\right)\right) d w(t), \\
x(t)= & \varnothing(t), \quad[-\tau, 0]
\end{aligned}
$$

is asymptotically stable in probability [1] for all $\tau>0$, then stochastic time-delay differential system (2.1) is called locally robustly stabilizable. If (2.6) is robustly stable [2], that is, the equilibrium point of (2.6) is asymptotically stable in the large [1] for all $\tau>0,(2.1)$ is globally robustly stabilizable.

Remark 2.3. Definition 2.2 gives locally (globally) robustly stabilizable of stochastic timedelay systems via memory state feedback control law $u(t)=K_{1} x(t)+K_{2} x(t-\tau)$, which is more general than that via memoryless state feedback control law [12]. This is because Definition 2.2 reduces to the corresponding definition under memoryless state feedback control law when $K_{2}=0$.

The aim of this paper is to find a constant memory state feedback control law (2.5), such that the equilibrium point of (2.6) is asymptotically stable in probability for all $\tau>0$.

\section{Main Results}

In this section, we will give some sufficient conditions of the stabilization of system (2.1). Without loss of generality, we can give the following assumption for nonlinear function $H_{i}$.

Assumption 3.1. There exists an $\epsilon>0$, such that

$$
\sup \left\|H_{i}\left(x, y, K_{1} x+K_{2} y\right)\right\| \leq \epsilon(\|x\|+\|y\|), \quad i=0,1,
$$

holds for all $x, y \in \mathbf{U}$ (a neighborhood of the origin).

The following general theorem is presented, which yields several applicable corollaries.

Theorem 3.2. If (3.1) holds and $K_{1}, K_{2} \in \mathbf{R}^{m \times n}, P>0, Q>0$ are the solutions of the following matrix inequality

$$
Z+Z_{1}<0
$$


then system (2.1) can be locally robustly stabilized by (2.5). If $\mathbf{U}$ is replaced by $\mathbf{R}^{n}$, then system (2.1) can be globally robustly stabilized by the same controller.

In (3.2), $Z$ and $Z_{1}$ are defined by

$$
\begin{gathered}
Z=\left[\begin{array}{cc}
\Sigma_{1} & \Sigma_{2} \\
* & \left(D+D_{1} K_{2}\right)^{\prime} P\left(D+D_{1} K_{2}\right)-Q
\end{array}\right], \\
Z_{1}=\left[\begin{array}{cc}
\Sigma_{3} & 0 \\
0 & \Sigma_{4}
\end{array}\right]
\end{gathered}
$$

where

$$
\begin{gathered}
\Sigma_{1}=P\left(A+B_{1} K_{1}\right)+\left(A+B_{1} K_{1}\right)^{\prime} P+Q+\left(C+D_{1} K_{1}\right)^{\prime} P\left(C+D_{1} K_{1}\right), \\
\Sigma_{2}=P\left(B+B_{1} K_{2}\right)+\left(C+D_{1} K_{1}\right)^{\prime} P\left(D+D_{1} K_{2}\right), \\
\Sigma_{3}=\epsilon\left(3+3\|C\|+3\left\|D_{1}\right\| \cdot\left\|K_{1}\right\|+3 \epsilon+\|D\|+\epsilon\left\|D_{1}\right\| \cdot\left\|K_{2}\right\|\right)\|P\| I, \\
\Sigma_{4}=\epsilon\left(3+\|D\|+\left\|D_{1}\right\| \cdot\left\|K_{2}\right\|+\|C\|+\left\|D_{1}\right\| \cdot\left\|K_{1}\right\|+2 \epsilon\right)\|P\| I .
\end{gathered}
$$

Proof. Choose the following Lyapunov-Krasoviskii functional:

$$
V(t, x)=x^{\prime}(t) P x(t)+\int_{0}^{\tau} x^{\prime}(t-s) Q x(t-s) d s
$$

where $P>0$ and $Q>0$ are the solutions of (3.2). Let $\mathcal{L}$ be the infinitesimal generator of the closed-loop system (2.6), then, by the Itô's formula, we have

$$
\begin{aligned}
\mathcal{L}(t, x(t))= & x^{\prime}(t)\left[P\left(A+B_{1} K_{1}\right)+\left(A+B_{1} K_{1}\right)^{\prime} P+Q+\left(C+D_{1} K_{1}\right)^{\prime} P\left(C+D_{1} K_{1}\right)\right] x(t) \\
& +2 x^{\prime}(t)\left[P\left(B+B_{1} K_{2}\right)+\left(C+D_{1} K_{1}\right)^{\prime} \cdot P\left(D+D_{1} K_{2}\right)\right] x(t-\tau)+x^{\prime}(t-\tau) \\
& \cdot\left[\left(D+D_{1} K_{2}\right)^{\prime} P\left(D+D_{1} K_{2}\right)-Q\right] x(t-\tau) \\
& +2 H_{0}^{\prime} P x(t)+2 H_{1}^{\prime} P\left(D+D_{1} K_{2}\right) x(t-\tau) \\
& +2 H_{1}^{\prime} P\left(C+D_{1} K_{1}\right) x(t)+H_{1}^{\prime} P H_{1} \\
= & {\left[\begin{array}{c}
x(t) \\
x(t-\tau)
\end{array}\right]^{\prime} Z\left[\begin{array}{c}
x(t) \\
x(t-\tau)
\end{array}\right]+2 H_{0}^{\prime} P x(t)+H_{1}^{\prime} P H_{1} } \\
& +2 H_{1}^{\prime} P\left(D+D_{1} K_{2}\right) x(t-\tau)+2 H_{1}^{\prime} P\left(C+D_{1} K_{1}\right) x(t) .
\end{aligned}
$$


In addition, by (3.1), we obtain

$$
\begin{aligned}
2 H_{0}^{\prime} P x & (t)+2 H_{1}^{\prime} P\left(D+D_{1} K_{2}\right) x(t-\tau)+2 H_{1}^{\prime} P\left(C+D_{1} K_{1}\right) x(t)+H_{1}^{\prime} P H_{1} \\
\leq & 2 \epsilon\|P\|\left(1+\|C\|+\left\|D_{1}\right\| \cdot\left\|K_{1}\right\|+\epsilon\right)\|x(t)\|^{2} \\
& +2 \epsilon\|P\|\left(1+\|C\|+\left\|D_{1}\right\| \cdot\left\|K_{1}\right\|+\|D\|+\left\|D_{1}\right\| \cdot\left\|K_{2}\right\|+\epsilon\right)\|x(t)\| \cdot\|x(t-\tau)\| \\
& +\left(2 \epsilon+\epsilon^{2}\right)\|P\| \cdot\|x(t-\tau)\|^{2} .
\end{aligned}
$$

By inequality $|a b| \leq(1 / 2)\left(a^{2}+b^{2}\right)$, then (3.7) becomes

$$
\begin{aligned}
& 2 H_{0}^{\prime} P x(t)+2 H_{1}^{\prime} P\left(D+D_{1} K_{2}\right) x(t-\tau)+2 H_{1}^{\prime} P\left(C+D_{1} K_{1}\right) x(t)+H_{1}^{\prime} P H_{1} \\
& \leq \epsilon\left(3+3\|C\|+3\left\|D_{1}\right\| \cdot\left\|K_{1}\right\|+3 \epsilon+\|D\|+\left\|D_{1}\right\| \cdot\left\|K_{2}\right\|\right)\|P\| I\|x(t)\|^{2} \\
&+\epsilon\left(3+\|D\|+\left\|D_{1}\right\| \cdot\left\|K_{2}\right\|+\left\|K_{2}\right\|\|C\|+\left\|D_{1}\right\| \cdot\left\|K_{1}\right\|+2 \epsilon\right)\|P\| I\|x(t-\tau)\|^{2} \\
&= {\left[\begin{array}{c}
x(t) \\
x(t-\tau)
\end{array}\right]^{\prime} Z_{1}\left[\begin{array}{c}
x(t) \\
x(t-\tau)
\end{array}\right] . }
\end{aligned}
$$

Substituting (3.8) into (3.6), it follows

$$
\mathcal{L} V(t, x(t)) \leq\left[\begin{array}{c}
x(t) \\
x(t-\tau)
\end{array}\right]^{\prime}\left(Z+Z_{1}\right)\left[\begin{array}{c}
x(t) \\
x(t-\tau)
\end{array}\right]
$$

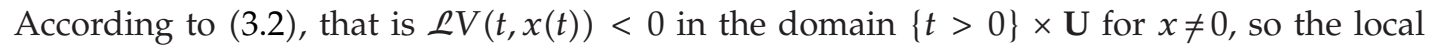
stabilization of Theorem 3.2 is obtained by Corollary of [1]. By the same discussion, the global stabilization conditions can be also obtained by Theorem 4.4 of [1].

From Theorem 3.2, we can derive some useful results, which can be expressed in terms of LMIs.

Corollary 3.3. If $H_{i} \equiv 0, i=0,1$, and the matrix inequality $Z<0$ has solutions $P>0, Q>0$ and $K_{1}, K_{2} \in \mathbf{R}^{m \times n}$, then the linear stochastic time-delay system

$$
d x(t)=\left(A x(t)+B x(t-\tau)+B_{1} u(t)\right) d t+\left(C x(t)+D x(t-\tau)+D_{1} u(t)\right) d w(t)
$$

is globally robustly stabilizable. If $D=0, D_{1}=0$, and the following LMI:

$$
\left[\begin{array}{cccc}
\Sigma_{5} & \widehat{P} & \widehat{P} C^{\prime} & B_{1} X \\
\widehat{P} & -\widehat{Q} & 0 & 0 \\
C \widehat{P} & 0 & -\widehat{P} & 0 \\
X B_{1}^{\prime} & 0 & 0 & -\widehat{Q}
\end{array}\right]<0
$$


has solutions $\widehat{P}>0, Y \in \mathbf{R}^{m \times n}, X \in \mathbf{R}^{n \times m}$, and $\widehat{Q}>0$, then

$$
d x(t)=\left(A x(t)+B x(t-\tau)+B_{1} u(t)\right) d t+C x(t) d w(t)
$$

is globally robustly stabilizable, where $\Sigma_{5}=A \widehat{P}+\widehat{P} A^{\prime}+B_{1} Y+Y^{\prime} B_{1}^{\prime}+B \widehat{Q} B^{\prime}+B X B_{1}^{\prime}+B_{1} X B^{\prime}$, and a stabilizing feedback control law $u(t)=K_{1} x(t)+K_{2} x(t-\tau)=Y \widehat{P}^{-1} x(t)+X \widehat{Q}^{-1} x(t-\tau)$.

Proof. If $H_{i}(\cdot, \cdot, \cdot)=0, i=0,1$, we can take $\epsilon=0$ in (3.1), then $\mathcal{L V}(t, x(t))<0$ for $(t, x) \in t>$ $0 \times \mathbf{R}^{n}$, except possibly at $x=0$.

Thus, the first part of Corollary 3.3 is proved.

Furthermore, if $D=0, D_{1}=0$, (3.2) degenerates into

$$
Z=\left[\begin{array}{cc}
\Sigma_{6} & P\left(B+B_{1} K_{2}\right) \\
\left(B+B_{1} K_{2}\right)^{\prime} P & -Q
\end{array}\right]<0,
$$

where $\Sigma_{6}=P\left(A+B_{1} K_{1}\right)+\left(A+B_{1} K_{1}\right)^{\prime} P+Q+C^{\prime} P C$.

According to Schur's complement, (3.13) is equivalent to

$$
\Sigma_{6}+P\left(B+B_{1} K_{2}\right) Q^{-1}\left(B+B_{1} K_{2}\right)^{\prime} P<0 .
$$

Then, pre- and post-(3.14) by $P^{-1}$, we have

$$
P^{-1} \Sigma_{6} P^{-1}+\left(B+B_{1} K_{2}\right) Q^{-1}\left(B+B_{1} K_{2}\right)^{\prime}<0 .
$$

Setting $\widehat{P}=P^{-1}, Y=K_{1} P^{-1}, \widehat{Q}=Q^{-1}$, and $X=K_{2} Q^{-1}$. Again, by Schur's complement, (3.15) is equivalent to (3.11). Thus, the second part of Corollary 3.3 is also proved.

Remark 3.4. Reference [13] considered the analogous problem to Corollary 3.3 by delay feedback, where the main result is expressed by means of generalized algebraic Riccati equations (GAREs) GAREs. However, Corollary 3.3 gives a sufficient condition in terms of LMIs which are easy to be solved.

Corollary 3.5. If the following LMI:

$$
\left[\begin{array}{cccccc}
\Gamma_{1}^{*} & \sqrt{2} \widehat{P} C^{\prime} & \widehat{P} & B^{\prime} & 0 & 0 \\
\sqrt{2} C \widehat{P} & -\widehat{P} & 0 & 0 & 0 & 0 \\
\widehat{P} & 0 & -I & 0 & 0 & 0 \\
B & 0 & 0 & -I & \sqrt{2} D^{\prime} & K_{2}^{\prime} B_{1}^{\prime} \\
0 & 0 & 0 & \sqrt{2} D & -\widehat{P} & 0 \\
0 & 0 & 0 & B_{1} K_{2} & 0 & -\widehat{P}
\end{array}\right]<0
$$


has solution $\widehat{P}>0, Y$, and $K_{2}$, then the stochastic linear time-delay controlled system

$$
d x(t)=\left(A x(t)+B x(t-\tau)+B_{1} u(t)\right) d t+\left(C x(t)+D x(t-\tau)+D_{1} u(t)\right) d w(t)
$$

is globally robustly stabilizable, where $\Gamma_{1}^{*}=A^{\prime} \widehat{P}+\widehat{P} A+B_{1} Y+Y^{\prime} B_{1}^{\prime}+\widehat{P}$. Moreover, the stabilizing feedback control law

$$
u(t)=Y \widehat{P}^{-1} x(t)+K_{2} x(t-\tau) .
$$

Proof. Applying the well-known inequality

$$
X^{\prime} Y+Y^{\prime} X \leq \gamma X^{\prime} X+\gamma^{-1} Y^{\prime} Y, \quad \forall \gamma>0,
$$

and supposing $\gamma=1$ for simplicity, we have

$$
\begin{aligned}
2 x^{\prime}(t) & P B_{1} K_{2} x(t-\tau)+2 x^{\prime}(t)\left(C+D_{1} K_{1}\right)^{\prime} P \cdot\left(D+D_{1} K_{2}\right) x(t-\tau) \\
\leq & x^{\prime}(t)\left[P+\left(C+D_{1} K_{1}\right)^{\prime} P\left(C+D_{1} K_{1}\right)\right] x(t) \\
& +x^{\prime}(t-\tau)\left[K_{2}^{\prime} B_{1}^{\prime} P B_{1} K_{2}+\left(D+D_{1} K_{2}\right)^{\prime} P \cdot\left(D+D_{1} K_{2}\right)\right] x(t-\tau) .
\end{aligned}
$$

Let $\Gamma_{1}=\Sigma_{1}+P+\left(C+D_{1} K_{1}\right)^{\prime} P\left(C+D_{1} K_{1}\right), \Gamma_{2}=2\left(D+D_{1} K_{2}\right)^{\prime} P\left(D+D_{1} K_{2}\right)+K_{2}^{\prime} B_{1}^{\prime} P B_{1} K_{2}-Q$. Then,

$$
Z \leq\left[\begin{array}{cc}
\Gamma_{1} & P B \\
* & \Gamma_{2}
\end{array}\right]=\Gamma
$$

Obviously, if $\Gamma<0$, then $Z<0$. Applying the Theorem 3.2, the closed-loop system of (3.17) is robustly stable [2].

Then, pre- and post-multiplying $\Gamma<0$ by $\operatorname{diag}\left\{P^{-1}, I\right\}$, and by Schur's complement, we have $\Gamma<0$ is equivalent to

$$
\left[\begin{array}{cccccc}
\Gamma_{1}^{*} & \sqrt{2} P^{-1} C^{\prime} & P^{-1} & B^{\prime} & 0 & 0 \\
\sqrt{2} C P^{-1} & -P^{-1} & 0 & 0 & 0 & 0 \\
P^{-1} & 0 & -Q^{-1} & 0 & 0 & 0 \\
B & 0 & 0 & -Q & \sqrt{2} D^{\prime} & K_{2}^{\prime} B_{1}^{\prime} \\
0 & 0 & 0 & \sqrt{2} D & -P^{-1} & 0 \\
0 & 0 & 0 & B_{1} K_{2} & 0 & -P^{-1}
\end{array}\right]<0
$$

where $\Gamma_{1}^{*}=A P^{-1}+P^{-1} A+B_{1} K_{1} P^{-1}+P^{-1} K_{1}^{\prime} B_{1}^{\prime}+P^{-1}$. Set $\widehat{P}=P^{-1}, Y=K_{1} P^{-1}=K_{1} \widehat{P}, Q=I$, (3.22) is equivalent to (3.16). This ends the proof of Corollary 3.5.

Below, for $D=0, D_{1}=0$, we give another sufficient condition for the local (global) stabilization of system (2.1) in the terms of LMIs. 
Theorem 3.6. For $D=0, D_{1}=0$ in (2.1), suppose (3.1) holds for all $x, y \in \mathbf{U}\left(x, y \in \mathbf{R}^{n}\right)$. If the LMIs:

$$
\begin{array}{ccccc}
{\left[\begin{array}{ccccc}
\Pi_{1} & \widehat{P} & \widehat{P} & \sqrt{2} \widehat{P} C^{\prime} & B+B_{1} K_{2} \\
\widehat{P} & -\widehat{Q} & 0 & 0 & 0 \\
\widehat{P} & 0 & -\frac{\alpha}{6 \epsilon^{2}} I & 0 & 0 \\
\sqrt{2} C \widehat{P} & 0 & 0 & -\widehat{P} & 0 \\
B^{\prime}+K_{2}^{\prime} B_{1}^{\prime} & 0 & 0 & 0 & -\epsilon^{2} I
\end{array}\right]<0} \\
\widehat{P} \leq \alpha I, \\
\widehat{Q} \leq \frac{\alpha I}{7 \epsilon^{2}} \\
0<\alpha \leq 1,
\end{array}
$$

have solutions $\widehat{P}>0, \alpha, \widehat{Q}>0, K_{2}$, and $Y \in \mathbf{R}^{m \times n}$, then system (2.1) can be locally (globally) robustly stabilized by

$$
u(t)=Y \widehat{P}^{-1} x(t)+K_{2} x(t-\tau),
$$

where $\Pi_{1}=A \widehat{P}+\widehat{P} A^{\prime}+B_{1} Y+Y^{\prime} B^{\prime}{ }_{1}+\widehat{P}$.

Proof. Applying the well-known inequality (3.19) again and supposing $\gamma=1$ for simplicity, we have (if $0<P \leq I / \alpha$ for some $\alpha>0$ )

$$
\begin{array}{rl}
2 H_{0}^{\prime} P & x(t)+2 H_{1}^{\prime} P C x(t)+H_{1}^{\prime} P H_{1} \\
& \leq \frac{6 \epsilon^{2}}{\alpha}\left(\|x(t)\|^{2}+\|x(t-\tau)\|^{2}\right)+x^{\prime}(t)\left(P+C^{\prime} P C\right) x(t)
\end{array}
$$

which holds because

$$
\begin{aligned}
2 H_{0}^{\prime} P x & =H_{0}^{\prime} P^{1 / 2} \cdot P^{1 / 2} x+x^{\prime} P^{1 / 2} \cdot P^{1 / 2} H_{0}^{\prime} \\
\leq & H_{0}^{\prime} P H_{0}+x^{\prime} P x \\
\leq & \frac{2 \epsilon^{2}}{\alpha}\left(\|x(t)\|^{2}+\|x(t-\tau)\|^{2}\right)+x^{\prime} P x, \\
H_{1}^{\prime} P H_{1} \leq & \frac{2 \epsilon^{2}}{\alpha}\left(\|x(t)\|^{2}+\|x(t-\tau)\|^{2}\right), \\
2 H_{1}^{\prime} P C x= & H_{1}^{\prime} P^{1 / 2} \cdot P^{1 / 2} C x+x^{\prime} C^{\prime} P^{1 / 2} \cdot P^{1 / 2} H_{1}^{\prime} \\
\leq & \frac{2 \epsilon^{2}}{\alpha}\left(\|x(t)\|^{2}+\|x(t-\tau)\|^{2}\right) \\
& +x^{\prime}(t) C^{\prime} P C x(t) .
\end{aligned}
$$


Substituting (3.28) into (3.6), it follows that

$$
\mathcal{L}(t, x(t)) \leq\left[\begin{array}{c}
x(t) \\
x(t-\tau)
\end{array}\right]^{\prime} \hat{Z}\left[\begin{array}{c}
x(t) \\
x(t-\tau)
\end{array}\right]
$$

where

$$
\widehat{Z}=\left[\begin{array}{cc}
\Pi_{1}^{*}+\frac{6}{\alpha} \epsilon^{2} I & P\left(B+B_{1} K_{2}\right) \\
* & \frac{6}{\alpha} \epsilon^{2} I-Q
\end{array}\right] .
$$

Considering (3.24), (3.25), and (3.26), it follows that

$$
\widehat{Z} \leq\left[\begin{array}{cc}
\Pi_{1}^{*}+\frac{6}{\alpha} \epsilon^{2} I & P\left(B+B_{1} K_{2}\right) \\
* & -\epsilon^{2} I
\end{array}\right] .
$$

Let

$$
Z_{1}=\left[\begin{array}{cc}
\Pi_{1}^{*}+\frac{6}{\alpha} \epsilon^{2} I & P\left(B+B_{1} K_{2}\right) \\
* & -\epsilon^{2} I
\end{array}\right],
$$

where $\Pi_{1}^{*}=P\left(A+B_{1} K_{1}\right)+\left(A+B_{1} K_{1}\right)^{\prime} P+Q+P+2 C^{\prime} P C$.

Obviously, if $Z_{1}<0$, then $\hat{Z}<0$. So if (3.1) holds for all $x \in \mathbf{U}\left(x \in \mathbf{R}^{n}\right)$, and $\hat{Z}<0$, then system (2.1) can be locally (globally) robustly stabilized by $u(t)=K_{1} x(t)+K_{2} x(t-\tau)$.

Note that $Z_{1}<0$ is equivalent to that

$$
P\left(A+B_{1} K_{1}\right)+\left(A+B_{1} K_{1}\right)^{\prime} P+Q+P+2 C^{\prime} P C+\frac{6}{\alpha} \epsilon^{2} I+P\left(B+B_{1} K_{2}\right) \epsilon^{-2} I\left(K_{2}^{\prime} B_{1}^{\prime}+B^{\prime}\right) P<0 .
$$

Then pre- and postmultiply (3.34) by $P^{-1}$, we have

$$
\begin{aligned}
(A & \left.+B_{1} K_{1}\right) P^{-1}+P^{-1}\left(A+B_{1} K_{1}\right)^{\prime}+P^{-1} Q P^{-1} \\
& +P^{-1}+2 P^{-1} C^{\prime} P C P^{-1}+P^{-1} \frac{6}{\alpha} \epsilon^{2} I P^{-1} \\
& +\left(B+B_{1} K_{2}\right) \epsilon^{-2} I\left(K_{2}^{\prime} B_{1}^{\prime}+B^{\prime}\right)<0 .
\end{aligned}
$$

Setting $\widehat{P}=P^{-1}, Y=K_{1} P^{-1}=K_{1} \widehat{P}$, and $\widehat{Q}=Q^{-1}$ by the Schur's complement, (3.35) is equivalent to (3.23). Thus, the theorem is proved.

In the special case when $K_{2}=0$, our results reduce the corresponding results in memoryless state feedback case. The following theorem gives a sufficient condition for the existence of memoryless state feedback controller of system (2.1) with $D=0, D_{1}=0$. 
Theorem 3.7. For $D=0, D_{1}=0$ in (2.1), suppose there exists an $\epsilon>0$,

$$
\sup \left\|H_{i}\left(x, y, K_{1} x\right)\right\| \leq \epsilon(\|x\|+\|y\|), \quad i=0,1,
$$

holds for all $x, y \in \mathbf{U}\left(x, y \in \mathbf{R}^{n}\right)$, if the LMIs

$$
\left[\begin{array}{ccccc}
\Pi_{1} & \widehat{P} & \widehat{P} & \sqrt{2} \widehat{P} C^{\prime} & B \\
\widehat{P} & -\widehat{Q} & 0 & 0 & 0 \\
\widehat{P} & 0 & -\frac{\alpha}{6 \epsilon^{2}} I & 0 & 0 \\
\sqrt{2} C \widehat{P} & 0 & 0 & -\widehat{P} & 0 \\
B^{\prime} & 0 & 0 & 0 & -\epsilon^{2} I
\end{array}\right]<0
$$

and (3.24), (3.25), and (3.26) have solutions $\widehat{P}>0, \widehat{Q}>0, \alpha$, and $Y \in \mathbf{R}^{m \times n}$, then system (2.1) can be locally(globally) robustly stabilized by

$$
u(t)=Y \widehat{P}^{-1} x(t)
$$

Proof. It is derived by the same procedure as the proof of Theorem 3.6.

By the above discussion about stochastic systems with single delay (2.1), we further study robust stabilization for the following stochastic systems with multiple delays

$$
\begin{aligned}
d x(t)= & {\left[A x(t)+\sum_{j=1}^{q} B_{j} x\left(t-\tau_{j}\right)+\sum_{j=1}^{q} B_{1 j} u_{j}(t)+H_{0}\left(x(t), x\left(t-\tau_{j}\right), u_{j}(t)\right)\right] d t } \\
& +\left[C x(t)+\sum_{j=1}^{q} D_{j} x\left(t-\tau_{j}\right)+\sum_{j=1}^{q} D_{1 j} u_{j}(t)+H_{1}\left(x(t), x\left(t-\tau_{j}\right), u_{j}(t)\right)\right] d w(t), \\
x(t)= & \varnothing(t) \in L^{2}\left(\omega, \mathcal{F}_{0}, C\left([-h, 0], R^{n}\right)\right), \quad t \in[-h, 0],
\end{aligned}
$$

where $\tau_{j}>0, j=1, \ldots, q$, denote the state delay; $h=\max \left\{\tau_{j}, j \in[1, q]\right\}$.

For system (3.39), the following memory state feedback control law is adopted:

$$
u_{j}(t)=K_{j 1} x(t)+K_{j 2} x\left(t-\tau_{j}\right), \quad j=1, \ldots, q .
$$


Applying control law (3.40) to system (3.39), the resulting closed-loop system is given by

$$
\begin{aligned}
d x(t)= & {\left[\bar{A} x(t)+\sum_{j=1}^{q} \bar{B} x\left(t-\tau_{j}\right)+H_{0}\left(x(t), x\left(t-\tau_{j}\right), K_{j 1} x(t)+K_{j 2} x\left(t-\tau_{j}\right)\right)\right] d t } \\
& +\left[\bar{C} x(t)+\sum_{j=1}^{q} \bar{D} x\left(t-\tau_{j}\right)+H_{1}\left(x(t), x\left(t-\tau_{j}\right), K_{j 1} x(t)+K_{j 2} x\left(t-\tau_{j}\right)\right)\right] d w(t), \\
x(t)= & \varnothing(t) \in L^{2}\left(\omega, \mathcal{F}_{0}, C\left([-h, 0], R^{n}\right)\right), \quad t \in[-h, 0],
\end{aligned}
$$

where $\bar{A}=A+\sum_{j=1}^{q} B_{1 j} K_{j 1}, \bar{B}=B_{j}+B_{1 j} K_{j 2}, \bar{C}=C+\sum_{j=1}^{q} D_{1 j} K_{j 1}, \bar{D}=D_{j}+D_{1 j} K_{j 2}$.

By the same analysis as Theorem 3.2, we obtain the following theorem which gives a general sufficient condition for the robust stabilization of stochastic multiple time-delays system (3.39).

Theorem 3.8. If (3.1) holds, and $K_{j 1}, K_{j 2}, P>0$, and $Q>0$ are the solutions of the following matrix inequality

$$
Z^{0}+Z^{1}<0
$$

then system (3.39) can be locally robustly stabilized by $u_{j}(t)=K_{j 1} x(t)+K_{j 2} x\left(t-\tau_{j}\right)$. Especially if $\mathbf{U}$ is replaced by $\mathbf{R}^{n}$, then system (3.39) can be globally robustly stabilized by the same controller.

In (3.42), $Z^{0}$ and $Z^{1}$ are defined by

$$
\begin{aligned}
& Z^{0}=\left[\begin{array}{cc}
Z_{11}^{0} & Z_{12}^{0} \\
* & Z_{22}^{0}
\end{array}\right], \\
& Z^{1}=\left[\begin{array}{cc}
Z_{11}^{1} & 0 \\
0 & Z_{22}^{1}
\end{array}\right],
\end{aligned}
$$

where

$$
\begin{aligned}
Z_{11}^{0}= & P\left(A+\sum_{j=1}^{q} B_{1 j} K_{j 1}\right)+\left(A+\sum_{j=1}^{q} B_{1 j} K_{j 1}\right)^{\prime} P+Q \\
& +\left(C+\sum_{j=1}^{q} D_{1 j} K_{j 1}\right)^{\prime} P\left(C+\sum_{j=1}^{q} D_{1 j} K_{j 1}\right), \\
Z_{12}^{0}= & P \sum_{j=1}^{q}\left(B_{j}+B_{1 j} K_{j 2}\right)+\left(C+\sum_{j=1}^{q} D_{1 j} K_{j 1}\right)^{\prime} P \sum_{j=1}^{q}\left(D_{j}+D_{1 j} K_{j 2}\right),
\end{aligned}
$$


Mathematical Problems in Engineering

$$
\begin{gathered}
Z_{22}^{0}=\left[\sum_{j=1}^{q}\left(D_{j}+D_{1 j} K_{j 2}\right)\right]^{\prime}\left[\sum_{j=1}^{q}\left(D_{j}+D_{1 j} K_{j 2}\right)\right]-Q, \\
Z_{11}^{1}=\epsilon\left(3+3\|C\|+3\left\|\sum_{j=1}^{q} D_{1 j}\right\| \cdot\left\|\sum_{j=1}^{q} K_{j 1}\right\|+3 \epsilon\right. \\
\left.+\|D\|+\epsilon\left\|\sum_{j=1}^{q} D_{1 j}\right\| \cdot\left\|\sum_{j=1}^{q} K_{j 2}\right\|\right)\|P\| I, \\
Z_{22}^{1}=\epsilon\left(3+\|D\|+\left\|\sum_{j=1}^{q} D_{1 j}\right\| \cdot\left\|\sum_{j=1}^{q} K_{j 2}\right\|+\|C\|\right. \\
\left.+\left\|\sum_{j=1}^{q} D_{1 j}\right\| \cdot\left\|\sum_{j=1}^{q} K_{j 1}\right\|+2 \epsilon\right)\|P\| I .
\end{gathered}
$$

Remark 3.9. From Theorem 3.7, some useful results can be easily derived for stochastic multiple timedelays systems (3.39), which are similar to the results obtained for stochastic single time-delay systems (2.1).

\section{Numerical Example}

Now, we present one example to illustrate the effectiveness of our developed result (Theorem 3.6) in testing the stabilization of nonlinear stochastic time-delay system (2.1).

In (2.1), take $D=0, D_{1}=0$, and

$$
\begin{gathered}
A=\left[\begin{array}{ll}
-5.00 & 2.23 \\
-1.56 & 2.15
\end{array}\right], \quad B=\left[\begin{array}{cc}
-0.24 & 0.89 \\
1.22 & -0.76
\end{array}\right], \\
B_{1}=\left[\begin{array}{c}
-2.25 \\
4.48
\end{array}\right], \quad C=\left[\begin{array}{cc}
-0.05 & -0.15 \\
0.15 & -0.10
\end{array}\right], \\
H_{0}=\left[\begin{array}{l}
\sin \left(u(t) x_{2}(t-\tau)\right) x_{1}(t) \\
\cos \left(u(t) x_{1}(t-\tau)\right) x_{2}(t)
\end{array}\right], \\
H_{1}=\left[\begin{array}{c}
\exp \left(-\left(u(t)+x_{1}(t-\tau)+x_{2}(t-\tau)\right)^{2}\right) x_{2}(t) \\
\exp \left(-\left(u^{2}(t) x_{1}^{2}(t-\tau)\right)\right) x_{1}(t)
\end{array}\right], \\
\phi(0)=\left[\begin{array}{ll}
10 & 8
\end{array}\right]^{\prime}, \quad \tau=0.5 .
\end{gathered}
$$

Obviously, (2.1) holds for all $x \in \mathbf{R}^{n}$ with $\epsilon=1$. 
Case 1 (Memory State Feedback Stabilization). Substituting all the above data into (3.23) and then solving the LMIs (3.23), (3.24), (3.25), and (3.26) by LMIs Toolbox, we can obtain solutions

$$
\begin{gathered}
\widehat{P}=\left[\begin{array}{cc}
0.4625 & -0.0626 \\
-0.0626 & 0.3383
\end{array}\right]>0 \\
\alpha=0.9015 \\
Y=\left[\begin{array}{ll}
-0.1377 & -0.6674
\end{array}\right] \\
K_{2}=\left[\begin{array}{ll}
-0.2391 & 0.2149
\end{array}\right] \\
\widehat{Q}=\left[\begin{array}{ll}
0.1141 & 0.0052 \\
0.0052 & 0.0974
\end{array}\right]>0
\end{gathered}
$$

So by Theorem 3.6, system (2.1) can be globally robustly stabilized by

$$
\begin{aligned}
u(t)= & \Upsilon \widehat{P}^{-1} x(t)+K_{2} x(t-\tau) \\
= & -0.5790 x_{1}(t)-2.0795 x_{2}(t) \\
& -0.2391 x_{1}(t-\tau)+0.2149 x_{2}(t-\tau) .
\end{aligned}
$$

The state trajectories of close-loop system (2.6) and control curve in memory state feedback case are illustrated as Figure 1, from which, we see that the closed-loop system (2.6) takes only one second to have been stable.

Case 2 (Memory-Less State Feedback Stabilization). Solving LMIs (3.37), (3.24), (3.25), and (3.26), we obtain

$$
\begin{gathered}
\widehat{P}=\left[\begin{array}{cc}
0.5609 & -0.1964 \\
-0.1964 & 0.4117
\end{array}\right]>0, \\
Y=\left[\begin{array}{ll}
0.0602 & -0.5168
\end{array}\right] \\
\widehat{Q}=\left[\begin{array}{cc}
0.0923 & 0.0017 \\
0.0017 & 0.0790
\end{array}\right]>0 \\
\alpha=0.9243
\end{gathered}
$$

So by Theorem 3.7, system (2.1) can be globally robustly stabilized by

$$
u(t)=Y \widehat{P}^{-1} x(t)=-0.4449 x_{1}(t)-1.5770 x_{2}(t) .
$$




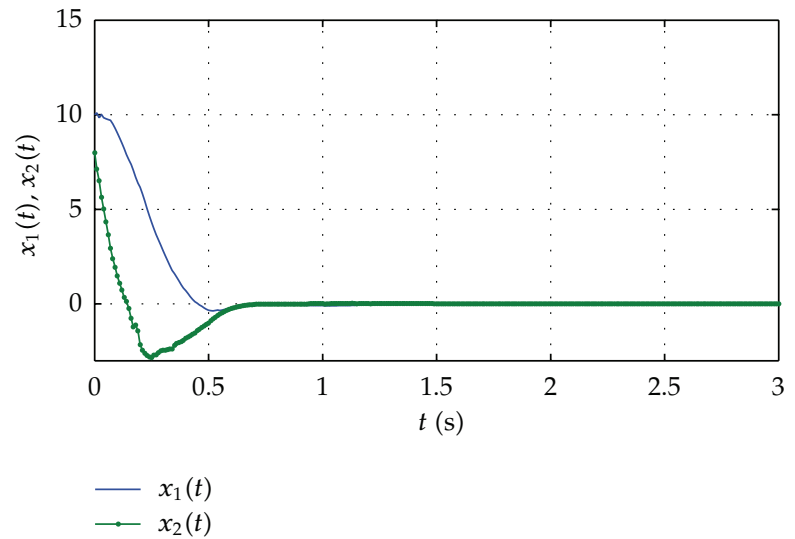

(a)

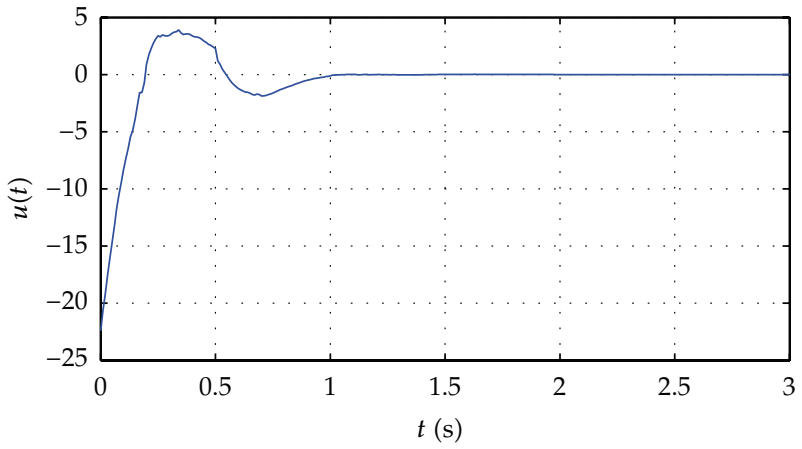

(b)

Figure 1: State trajectories and control input in memory state feedback case.

The state trajectories of close-loop system (2.6) and control curve in memoryless state feedback case are illustrated as Figure 2, from which, it can be seen that the closed-loop system (2.6) takes 1.5 seconds to have been stable.

From the two simulation results, the time needed to stabilize system using memory state feedback controller is less than that using memory-less state feedback controller, which shows the advantage of memory state feedback control.

\section{Conclusions}

This paper has discussed memory state feedback stabilization of stochastic systems with time-delay and nonlinear uncertainties. Some sufficient conditions have been given for the existence of a memory state feedback stabilizing control law in terms of linear matrix inequalities, which have the advantage of easy computation. The corresponding results to stochastic single time-delay systems have been further extended to the stochastic multiple time-delays systems. The results obtained in this paper can be reduced to the corresponding results in memoryless state feedback case and may also be extended to other stochastic system model. 


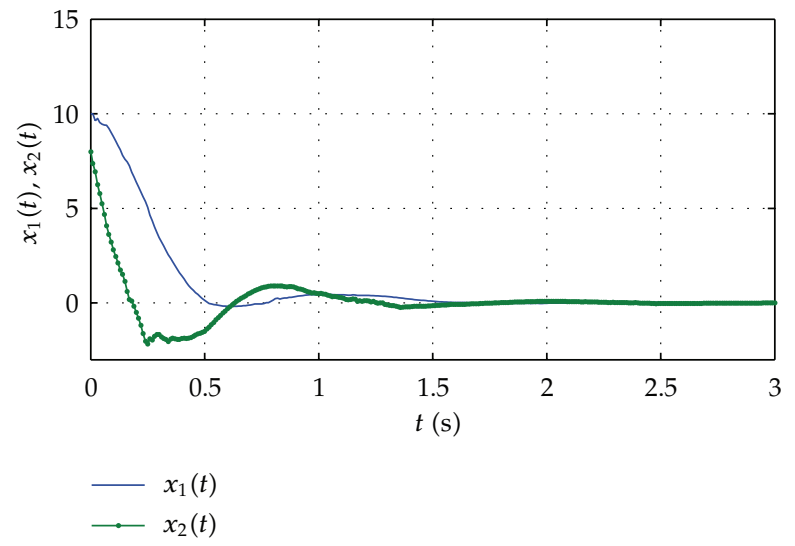

(a)

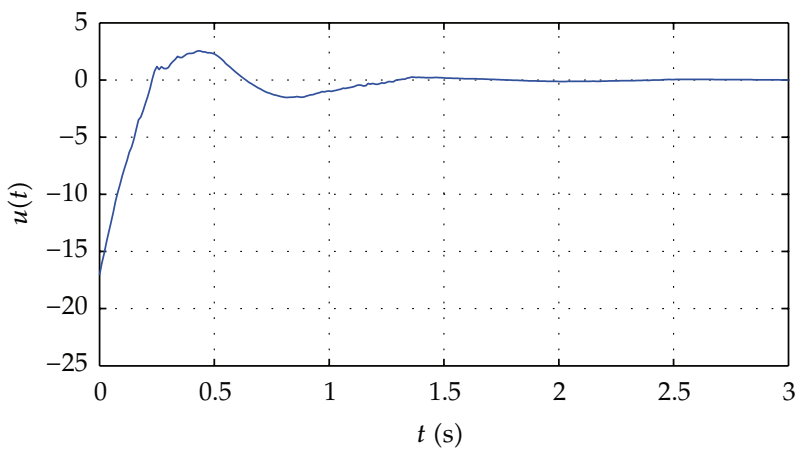

(b)

Figure 2: State trajectories and control input in memoryless state feedback case.

\section{Acknowledgment}

This work is supported by the National Natural Science Foundation of China under Grant 61074088, and the Starting Research Foundation of Shandong Polytechnic University under Grant 12045501. The authors thank the reviewers and editors for their very helpful comments and suggestions which have improved the presentation of this paper.

\section{References}

[1] R. Z. Has'minskii, Stability of Differential Equations[M], Sijtioff and Noordhoff, Alphen, The Netherlands, 1980.

[2] E. I. Verriest and P. Florchinger, "Stability of stochastic systems with uncertain time delays," Systems and Control Letters, vol. 24, no. 1, pp. 41-47, 1995.

[3] J. Li, W. Zhang, and Y. Mu, "Stability of nonlinear stochastic systems with uncertain time delays," in Proceedings of the 7th World Congress on Intelligent Control and Automation (WCICA '08), pp. 5540-5544, June 2008.

[4] C. Y. Lu, T. J. Su, and J. S. H. Tsai, “On robust stabilization of uncertain stochastic time-delay systemsan LMI-based approach," Journal of the Franklin Institute, vol. 342, no. 5, pp. 473-487, 2005.

[5] J. Zhang, P. Shi, and H. Yang, "Non-fragile robust stabilization and Hoo control for uncertain stochastic nonlinear time-delay systems," Chaos, Solitons and Fractals, vol. 42, no. 5, pp. 3187-3196, 2009. 
[6] Y. Zhang, Y. He, and M. Wu, "Delay-dependent robust stability for uncertain stochastic systems with interval time-varying delay," Acta Automatica Sinica, vol. 35, no. 5, pp. 577-582, 2009.

[7] X. Mao, "Robustness of stability of nonlinear systems with stochastic delay perturbations," Systems and Control Letters, vol. 19, no. 5, pp. 391-400, 1992.

[8] X. Mao, "Robustness of exponential stability of stochastic differential delay equations," IEEE Transactions on Automatic Control, vol. 41, no. 3, pp. 442-447, 1996.

[9] X. Mao, N. Koroleva, and A. Rodkina, "Robust stability of uncertain stochastic differential delay equations," Systems and Control Letters, vol. 35, no. 5, pp. 325-336, 1998.

[10] Y. Fu, Z. Tian, and S. Shi, "Output feedback stabilization for a class of stochastic time-delay nonlinear systems," IEEE Transactions on Automatic Control, vol. 50, no. 6, pp. 847-851, 2005.

[11] W. Zhang, B. S. Chen, and Q. Li, "Feedback stabilization of nonlinear stochastic time-delay systems with state and control-dependent noise," in Proceedings of the American Control Conference, pp. 3689 3692, July 2004.

[12] W. Zhang, X. Liu, S. Kong, and Q. Li, “On stabilization for a class of nonlinear stochastic time-delay systems: a matrix inequality approach[J]," Journal of Control Theory and Application, vol. 3, pp. 229-234, 2006.

[13] P. Florchinger and E. I. Verriest, "Stabilization of nonlinear stochastic systems with delay feedback," in Proceedings of the 32nd Conference on Decision and Control, pp. 859-860, December 1993.

[14] D. Yue and S. Won, "Delay-dependent robust stability of stochastic systems with time delay and nonlinear uncertainties," Electronics Letters, vol. 37, no. 15, pp. 992-993, 2001.

[15] J. P. Richard, "Time-delay systems: an overview of some recent advances and open problems," Automatica, vol. 39, no. 10, pp. 1667-1694, 2003.

[16] W. H. Chen, Z. H. Guan, and X. Lu, "Delay-dependent exponential stability of uncertain stochastic systems with multiple delays: An LMI approach," Systems and Control Letters, vol. 54, no. 6, pp. 547$555,2005$.

[17] S. Xu, P. Shi, Y. Chu, and Y. Zou, "Robust stochastic stabilization and Ho control of uncertain neutral stochastic time-delay systems," Journal of Mathematical Analysis and Applications, vol. 314, no. 1, pp. $1-16,2006$.

[18] W. Zhang, G. Feng, and Q. Li, "Robust $H_{\infty}$ filtering for nonlinear stochastic state-delayed systems," in Proceedings of the 7th World Congress on Intelligent Control and Automation (WCICA '08), pp. 2212-2217, June 2008.

[19] D. W. Ross, "Controller design for time lag systems via a quadratic criterion," IEEE Transactions on Automatic Control, vol. 16, no. 6, pp. 664-672, 1971.

[20] Y. S. Moon, P. Park, W. H. Kwon, and Y. S. Lee, “Delay-dependent robust stabilization of uncertain state-delayed systems," International Journal of Control, vol. 74, no. 14, pp. 1447-1455, 2001.

[21] B. Chen, J. Lam, and S. Xu, "Memory state feedback guaranteed cost control for neutral delay systems[J]," Internation Journal of Innovative Computing, Information, Control, vol. 2, pp. 293-303, 2006.

[22] Z. H. Jiang, W. H. Gui, Y. F. Xie, and C. H. Yang, "Memory state feedback control for singular systems with multiple internal incommensurate constant point delays," Acta Automatica Sinica, vol. 35, no. 2, pp. 174-179, 2009.

[23] J. Xiong and J. Lam, "Stabilization of discrete-time Markovian jump linear systems via time-delayed controllers," Automatica, vol. 42, no. 5, pp. 747-753, 2006. 


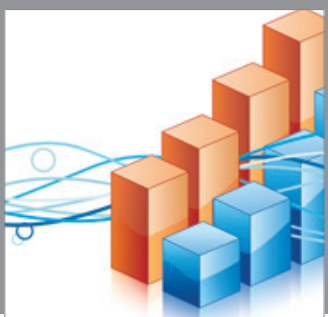

Advances in

Operations Research

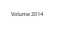

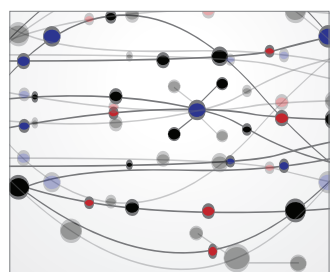

\section{The Scientific} World Journal
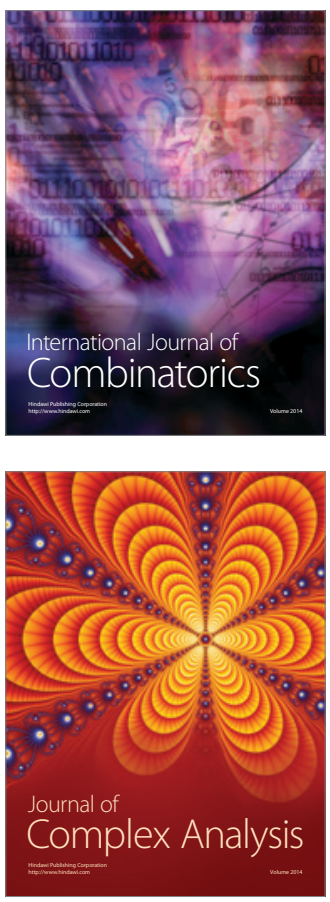

International Journal of

Mathematics and

Mathematical

Sciences
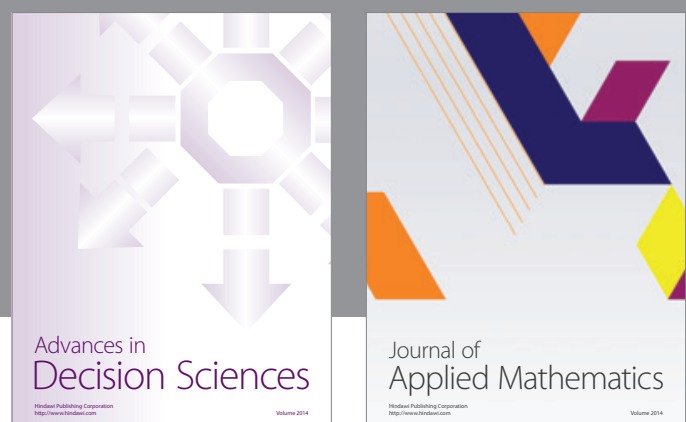

Journal of

Applied Mathematics
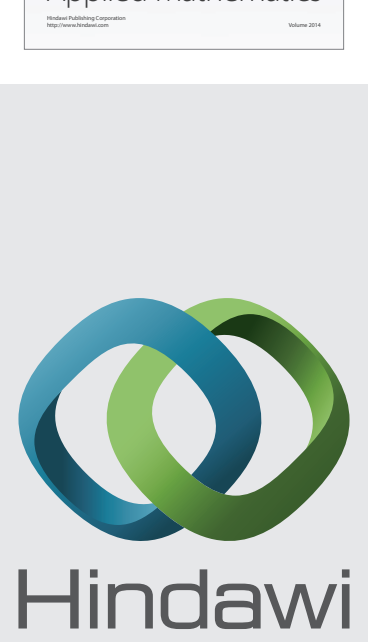

Submit your manuscripts at http://www.hindawi.com
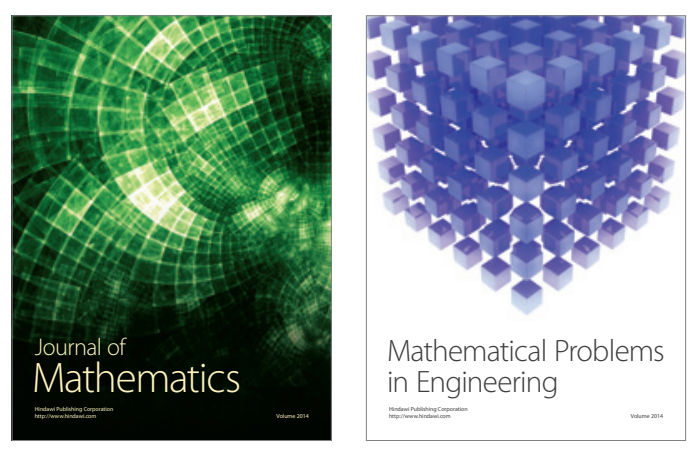

Mathematical Problems in Engineering
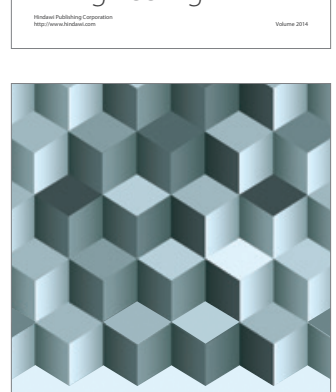

Journal of

Function Spaces
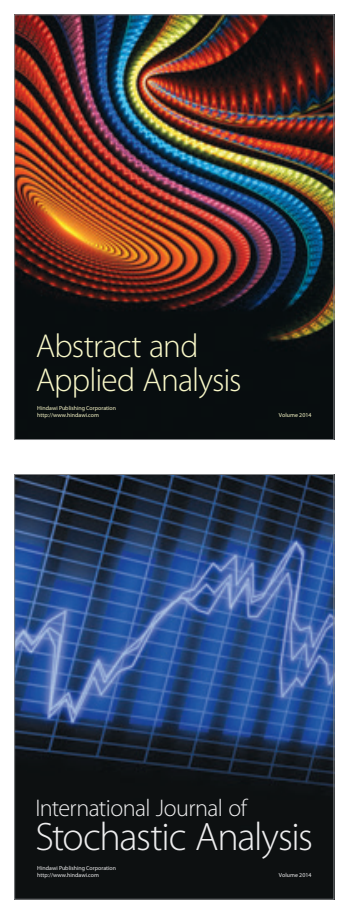

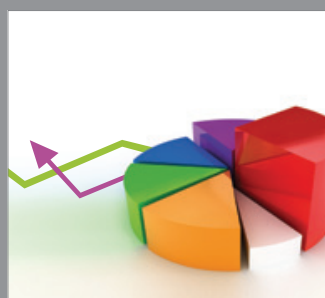

ournal of

Probability and Statistics

Promensencen
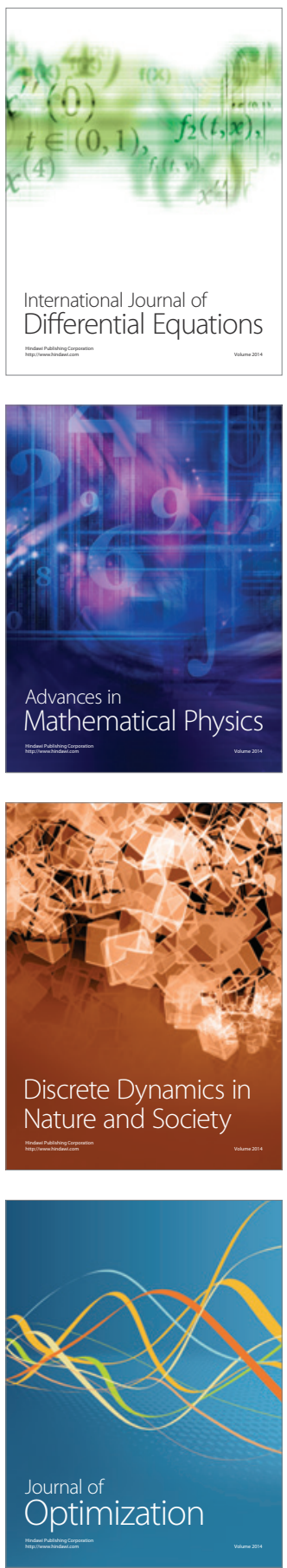